\title{
The initial stage of dam-break flow
}

\author{
Alexander Korobkin • Oguz Yilmaz
}

Received: 28 May 2007 / Accepted: 3 July 2008 / Published online: 25 July 2008

(C) Springer Science+Business Media B.V. 2008

\begin{abstract}
The liquid flow and the free surface shape during the initial stage of dam breaking are investigated. The method of matched asymptotic expansions is used to derive the leading-order uniform solution of the classical dam-break problem. The asymptotic analysis is performed with respect to a small parameter which characterizes the short duration of the stage under consideration. The second-order outer solution is obtained in the main flow region. This solution is not valid in a small vicinity of the intersection point between the initially vertical free surface and the horizontal rigid bottom. The dimension of this vicinity is estimated with the help of a local analysis of the outer solution close to the intersection point. Stretched local coordinates are used in this vicinity to resolve the flow singularity and to derive the leading-order inner solution, which describes the formation of the jet flow along the bottom. It is shown that the inner solution is self-similar and the corresponding boundary-value problem can be reduced to the well-known Cauchy-Poisson problem for water waves generated by a given pressure distribution along the free surface. An analysis of the inner solution reveals the complex shape of the jet head, which would be difficult to simulate numerically. The asymptotic solution obtained is expected to be helpful in the analysis of developed gravity-driven flows.
\end{abstract}

Keywords Asymptotic analysis · Dam break $\cdot$ Free-surface flows $\cdot$ Matched asymptotic expansions

\section{Introduction}

We consider the plane unsteady problem of gravity-driven flow, which is generated when a vertical dam in front of a liquid region is suddenly removed. The problem is treated in Eulerian coordinates. Initially the liquid is at rest and occupies the region $x^{\prime}>0,-H<y^{\prime}<0$ (see Fig. 1). Throughout a prime stands for dimensional variables and $H$ is the liquid depth. The part of the liquid boundary $x^{\prime}>0, y^{\prime}=0$ is the initial position of the liquid

A. Korobkin $(\varangle)$

School of Mathematics, University of East Anglia, Norwich NR4 7TJ, England

e-mail: a.korobkin@uea.ac.uk

A. Korobkin

Lavrentyev Institute of Hydrodynamics, 630090 Novosibirsk, Russia

O. Yilmaz

Department of Mathematics, Izmir Institute of Technology, 35430 Urla, Izmir, Turkey

e-mail:oguzyilmaz@iyte.edu.tr 


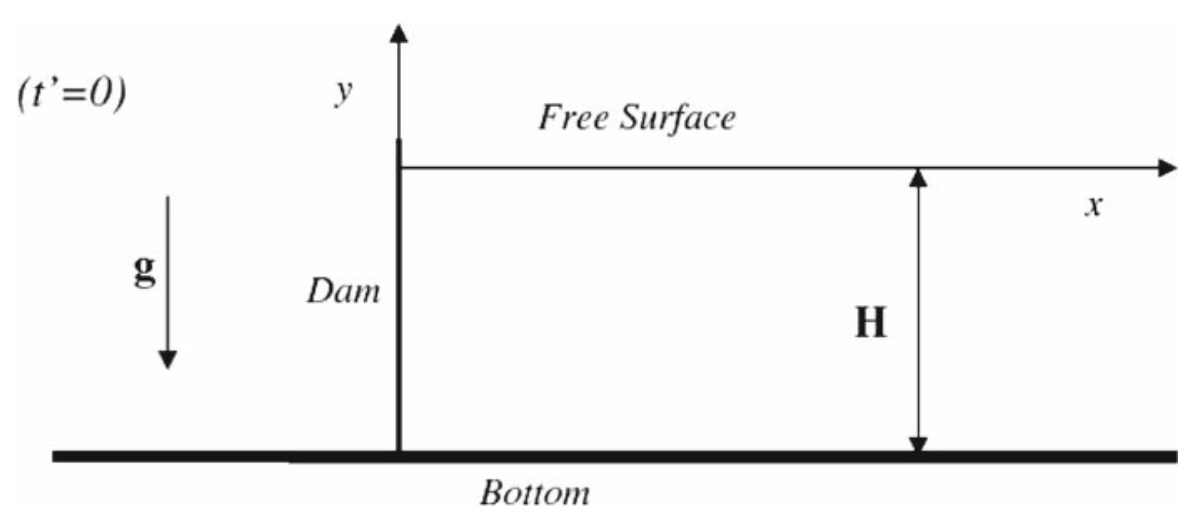

Fig. 1 Flow region at initial time instant $t^{\prime}=0$

free surface. The side wall, $x^{\prime}=0,-H<y^{\prime}<0$, represents a dam. The lower horizontal boundary, $y^{\prime}=-H$, represents the rigid bottom, which is dry in front of the dam, $x^{\prime}<0$. Initially the pressure distribution in the liquid is hydrostatic, $p^{\prime}\left(x^{\prime}, y^{\prime}, 0\right)=-\rho_{0} g y^{\prime}$, where $\rho_{0}$ is the liquid density and $g$ is the gravity acceleration. At the initial time instant, $t^{\prime}=0$, the dam is instantly removed and the gravity-driven flow starts. The liquid is assumed inviscid and incompressible. The resulting flow is potential and two-dimensional. We shall determine the liquid flow and the shape of its free surface during the early stages of the process.

Gravity-driven flows due to dam breaking were studied by Pohle [1] and Stoker [2] using the Lagrangian description. Pohle [1] wrote "Many hydrodynamic problems consider flows in which the region occupied by the fluid is a variable function of the time. The Euler representation is difficult to apply to such problems. The Lagrangian representation, however, has the far-reaching advantage that the independent space variables are the initial coordinates of the particles: the region occupied by the fluid is therefore a fixed region." This statement is true if there are no intersection points (lines) between the rigid boundaries and free surfaces of the liquid. However, this is not the case for many important problems of hydrodynamics including the water-entry problem, the dam-break problem and problems of floating or free-surface piercing bodies. This is due to the fact that close to the intersection points one needs to specify in advance which liquid particles originally belonging to the free surface may be found later on the rigid boundary and vice versa (see [3] for more detailed discussions). In other words, it is true that the flow region is fixed in the Lagrangian variables but the subdivision of the region boundary into "free surface" and "rigid boundary" is unknown in advance and has to be obtained as part of the solution. This makes the analysis of the dam-break problem in the Lagrangian variables not so attractive as it was expected by Pohle [1] and Stoker [2].

In [1], the liquid displacements and the hydrodynamic pressure are expanded in power series in the time $t^{\prime}$. Pohle presented a systematic procedure for the determination of the successive terms in these expansions. However, only the leading-order terms were constructed and analyzed. It is written in [1] that the calculated profile of the water surface for small times "can be expected to be a reasonable approximation to the physical problem except in the immediate neighborhood of the singular point", which is the point of the free-surface intersection with the rigid bottom. Figure 5 in [1] presents the water-surface profiles at different instants, but only at a distance above the bottom. Close to the bottom the small-time solution derived in the Lagrangian variables exhibits a non-physical shape of the water surface, with a part of it being below the bed (see Eq. III-14, p. 28 in [1]). A similar behaviour of the free surface close to the intersection point can be expected also with a small-time solution of the dam-break problem with Eulerian variables. This is because the Eulerian and Lagrangian variables are approximately the same within the small-time limit (see [3] for more details). In both Lagrangian and Eulerian descriptions the expansions of the solution in time power series should be considered as 'outer' solutions, which are needed to be corrected with 'inner' solutions near the intersection point. The outer and inner asymptotic solutions have to be matched in such a way as to arrive at a solution which is uniformly valid in the whole flow domain. We are unaware of attempts to construct the inner solutions of the dam-break problem either in Lagrangian or Eulerian variables. However, such an inner solution was successfully derived in a relevant problem concerning a uniformly accelerating wavemaker 
by King and Needham [4]. In the present study, we use the methodology and findings from [4], but we employ a formal small parameter $\varepsilon$, which characterizes the small duration of the initial stage under consideration.

There are numerous numerical and experimental studies on dam-break flows. Relevant to this study is the paper by Stansby et al. [5] in which the initial stage of dam-break flow, for dry- and wet-bed cases, is studied experimentally, and it is observed that for the dry-bed case a horizontal jet forms at small times. Stansby et al. also performed a numerical analysis of the dam-break problem. However, in order to avoid the singularity of the numerical solution at the intersection point, they had to pre-wet the bed in front of the dam by an artificial thin fluid layer. Several numerical studies performed during the past few years were based on the solution of nonlinear shallow-water equations using different methods such as the finite-volume method, the finite-difference method and so on (see [6-11]). There are very few asymptotic analyses of the dam-break problem. Hunt [12] used a kinematic wave approximation to obtain a closed-form solution for a sloping channel and mentioned that his solution is valid for large times.

The dam-break problem can be interpreted in the context of a liquid column collapsing under gravity [13]. In this problem a liquid column is initially at rest in the absence of the gravity. Gravity is "switched on" at $t^{\prime}=0$, then the liquid column starts to collapse and, at the same time, spreads out rapidly from the base, producing the phenomenon of the "base surge", which was observed in the atomic-bomb test at Bikini Lagoon. Penney and Thornhill [13] studied the collapse of a fluid column, which is surrounded by a lighter fluid. The analysis was performed in Eulerian variables both for small and moderate times. They derived the initial asymptotics of the solution for fluid columns of semi-cylindrical and hemispherical shapes, and showed that these asymptotics are not valid close to the base, where the fluid velocity is much higher than in the rest of the column.

The problem of a liquid-column collapse owing to sudden "switch on" of gravity is similar to the problem of a sudden upward motion of the bed towards the liquid. In a two-dimensional formulation the latter problem was studied by King and Needham [4] for a uniformly accelerating plate within a small-time approximation. King and Needham suggested an asymptotic procedure which allowed them to describe the initial development of a jet caused by the accelerating plate. In the present paper we use a similar asymptotic procedure, but we formally introduce a small parameter $\varepsilon$ instead of dealing with small-time expansions. This allows us to use classical methods of Cauchy-Poisson problems to derive the inner solution at leading order.

King and Needham [4] solved both the outer and inner problems to leading order as the non-dimensional time, $t^{\prime} \sqrt{H / g}$ in our notations, tends to zero. A consistent first-order solution was claimed. No second-order outer solution was given in [4], in spite of this being reasonably straightforward. In the present study we derive the second-order outer solution with the aim of determining both the size of the inner region and the far-field matching conditions required to obtain the inner potential problem. The same thing can be done by using the leading-order balance in the kinematic and dynamic boundary conditions together with the inner asymptotics of the leading-order outer solution, as done by King and Needham. However, it is hard to say that the problem of dam-break flow is fully identical to the problem successfully studied by King and Needham.

Asymptotic analysis of the potential problems with log-singularities is rather delicate. Needham, Billingham and King [14] studied the problem of the initial impulsive motion of a vertical plate and noted that this problem "has distinct structural differences, both mathematical and physical, from that of the initial uniformly accelerating motion of the plate, as described in King \& Needham (1994)". They derived the second-order outer solution close to the intersection form [14, Eq. 3.20]) and obtained the matching condition ((3.22) in [14]) in which the leading-order term contains contributions from both the first-order outer solution and second-order outer solution. The inner velocity potential [14, Eq. 3.28]) has the form which accounts for both the first- and second-order outer solutions.

One may argue that locally the dam-break flow close to the intersection point is independent of the liquid depth $H$ and is self-similar with respect to variables stretched with $g t^{\prime 2}$. Such a local flow is nonlinear and the position of the free surface should be obtained as part of the solution. This nonlinear inner flow was successfully matched to the leading-order outer dam-break flow in the case of an inclined dam, where the angle between the dam and the bed is less than $\pi / 2$ [15]. The matching was also successful in the problem of flow generated by the uniform acceleration of a rigid inclined plate [16]. However, for the vertical dam we were not able to match such a nonlinear and self-similar inner solution with the leading-order outer solution. We concluded that for the vertical dam-break flows the methodology of asymptotic analysis developed by King and Needham [4] indeed provides the uniformly 
valid initial solution. More discussions on the relation between our asymptotic procedure and that of King and Needham are given in the Conclusion.

\section{Formulation of the problem}

The gravity-driven flow caused by dam breaking is described in non-dimensional variables by the velocity potential $\varphi(x, y, t, \varepsilon)$. Non-dimensional unprimed variables are introduced as follows:

$x^{\prime}=H x, \quad y^{\prime}=H y, \quad t^{\prime}=T t$,

$\varphi^{\prime}=g H T \varphi, \quad p^{\prime}=\rho_{0} g H p, \quad T=\varepsilon^{1 / 2} \sqrt{H / g}$,

where the small non-dimensional parameter $\varepsilon$ is formal. This parameter is introduced in order to indicate that we are concerned here with the initial stage of the flow, in which $\varepsilon \rightarrow 0$.

The non-dimensional potential $\varphi(x, y, t, \varepsilon)$ satisfies the following equations

$\nabla^{2} \varphi=0 \quad($ in $\Omega(t))$,

$p=0 \quad($ on $\operatorname{FS}(t))$,

$\varphi_{y}=\varepsilon Y_{x} \varphi_{x}+Y_{t} \quad(y=\varepsilon Y(x, t, \varepsilon), x>0)$,

$\varphi_{x}=\varepsilon X_{y} \varphi_{y}+X_{t} \quad(x=\varepsilon X(y, t, \varepsilon),-1<y<\varepsilon Y(0, t, \varepsilon))$,

$\varphi_{y}=0 \quad(y=-1)$,

$-p=\varphi_{t}+\frac{\varepsilon}{2}|\nabla \varphi|^{2}+y \quad($ in $\Omega(t))$,

$\varphi(x, y, 0, \varepsilon)=0, \quad Y(x, 0, \varepsilon)=0, \quad X(y, 0, \varepsilon)=0 \quad(t=0)$,

$\varphi \rightarrow 0 \quad(x \rightarrow+\infty)$.

Here $\Omega(t)$ is the flow region and FS $(t)$ is the free surface of this region, which vary in time and have to be determined as part of the solution. The kinematic condition (4) is imposed on the upper part of the free surface, the shape of which is described by $y=\varepsilon Y(x, t, \varepsilon), x>0$. The kinematic condition (5) is imposed on the part of the free surface, which is initially vertical. The equation $x=\varepsilon X(y, t, \varepsilon)$ describes this part of the free surface. On both parts of the free surface the dynamic boundary condition (3) should be satisfied. The flow region $\Omega(t)$ in (2) is bounded by the free surface $\mathrm{FS}(t)$ and by the rigid bottom $y=-1$, along which the boundary condition (6) holds. Note that by definition

$X(\varepsilon Y(0, t, \varepsilon), t, \varepsilon)=0$.

The solution of the problem (2)-(9), as $\varepsilon \rightarrow 0$, is sought in the form

$$
\begin{aligned}
\varphi(x, y, t, \varepsilon) & =\varphi_{0}(x, y, t)+\varepsilon \varphi_{1}(x, y, t)+O\left(\varepsilon^{2}\right), \\
Y(x, t, \varepsilon) & =Y_{0}(x, t)+\varepsilon Y_{1}(x, t)+O\left(\varepsilon^{2}\right), \\
X(y, t, \varepsilon) & =X_{0}(y, t)+\varepsilon X_{1}(y, t)+O\left(\varepsilon^{2}\right) .
\end{aligned}
$$

Substituting the asymptotic expansions (10) in (2)-(9) and letting $\varepsilon \rightarrow 0$, we arrive at the following boundary-value problem for the leading-order velocity potential

$\nabla^{2} \varphi_{0}=0 \quad(x>0,-1<y<0)$,

$\varphi_{0}=0, \quad \frac{\partial Y_{0}}{\partial t}=\frac{\partial \varphi_{0}}{\partial y} \quad(x>0, y=0)$, 
$\varphi_{0}=-y t, \quad \frac{\partial X_{0}}{\partial t}=\frac{\partial \varphi_{0}}{\partial x} \quad(x=0,-1<y<0)$,

$\frac{\partial \varphi_{0}}{\partial y}=0 \quad(x>0, y=-1)$,

$\varphi_{0} \rightarrow 0 \quad(x \rightarrow+\infty)$.

The velocity potential $\varphi_{0}(x, y, t)$ is given as (see, for example, Pohle [1])

$\varphi_{0}(x, y, t)=\sum_{n=0}^{\infty} a_{n} \exp ^{-\sigma_{n} x} \sin \left[\sigma_{n} y\right], \quad \sigma_{n}=\frac{\pi}{2}(2 n+1), \quad a_{n}=2 t \sigma_{n}^{-2}(-1)^{n+1}$.

An analytical solution of the boundary-value problem (11) can also be obtained with the help of a conformal mapping and the theory of analytical functions (see [1]). However, for the purpose of this study the form of solution (12) is more convenient.

By algebra, the horizontal velocity component on the free surface $x=0,-1<y<0$ is

$\frac{\partial \varphi_{0}}{\partial x}(0, y, t)=\frac{2 t}{\pi} \log \left[\tan \frac{\pi}{4}(1+y)\right]$,

the vertical velocity component on the upper free surface, $x>0, y=0$, is

$\frac{\partial \varphi_{0}}{\partial y}(x, 0, t)=-\frac{4 t}{\pi} \arctan \left[\exp \left(\frac{-\pi x}{2}\right)\right]$

and the horizontal velocity along the bottom is

$\frac{\partial \varphi_{0}}{\partial x}(x,-1, t)=\frac{2 t}{\pi} \log \left[\tanh \frac{\pi x}{4}\right]$.

Correspondingly, the functions $X_{0}(y, t)$ and $Y_{0}(x, t)$ have the forms

$X_{0}(y, t)=\frac{1}{\pi} t^{2} \log \left[\tan \frac{\pi}{4}(1+y)\right]$,

$Y_{0}(x, t)=-\frac{2}{\pi} t^{2} \arctan \left[\exp \left(\frac{-\pi x}{2}\right)\right]$.

The velocity field given by the leading-order solution (12) is singular at the intersection point, $x=0, y=-1$, and coincides with the solution derived by Pohle [1].

In order to study the flow close to the intersection point, we introduce stretched local coordinates:

$x=a \xi, \quad y=-1+a \eta$,

where $a$ is a small positive constant, $a \ll 1$, and $\xi=O(1), \eta=O(1), t=O(1)$. At leading order as $a \rightarrow 0$ Eqs. 13 and 15 provide

$\frac{\partial \varphi_{0}}{\partial x}(0, y, t)=\frac{2 t}{\pi} \log \left[\frac{\pi}{4} a \eta\right]+O\left(a^{2}\right), \quad \frac{\partial \varphi_{0}}{\partial x}(x,-1, t)=\frac{2 t}{\pi} \log \left[\frac{\pi}{4} a \xi\right]+O\left(a^{2}\right)$.

It is convenient to introduce the complex potential

$w_{0}(z, t)=\varphi_{0}(x, y, t)+\mathrm{i} \psi_{0}(x, y, t), \quad z=x+\mathrm{i} y$,

where $\psi_{0}(x, y, t)$ is the stream function, and consider the leading-order complex velocity of the flow

$\frac{\mathrm{d} w_{0}}{\mathrm{~d} z}=\frac{\partial \varphi_{0}}{\partial x}-\mathrm{i} \frac{\partial \varphi_{0}}{\partial y}$.

Equations (18) provide

$\frac{\mathrm{d} w_{0}}{\mathrm{~d} z}=\frac{2 t}{\pi} \log \left[\frac{\pi}{4} a \zeta\right]+O\left(a^{2}\right), \quad \zeta=\xi+\mathrm{i} \eta$, 
where the branch of the logarithmic function is chosen such that $\log 1=0$. It can be checked that the asymptotic formula for the complex velocity potential (21) satisfies locally the boundary conditions in (11) and the asymptotic formulae (18). The boundary conditions in (11) give $w_{0}(-\mathrm{i}, t)=t$ at the intersection point. By integrating (21) in a small vicinity of this point, one gets

$w_{0}(z, t)=t+\frac{2 t}{\pi} a \log a \zeta+\frac{2 t}{\pi} a\left[\log \zeta+\log \frac{\pi}{4}-1\right] \zeta+O\left(a^{3}\right)$

as $a \rightarrow 0$ and $|\zeta|=O(1)$. The real part of the complex potential (22) provides the asymptotic behaviour of the leading-order velocity potential close to the intersection point as

$\varphi_{0}=t+\frac{2 t}{\pi} a \log a \xi+\frac{2 t}{\pi} a\left[\log \rho+\log \frac{\pi}{4}-1\right] \xi-\frac{2 t}{\pi} a \eta \theta(\xi, \eta)+O\left(a^{3}\right)$,

where $\xi=\rho \cos \theta$ and $\eta=\rho \sin \theta$.

Equations (13), (15) and (16) demonstrate that the formal asymptotic expansions (10) are not valid near the intersection point and, therefore, they should be considered as an "outer" asymptotic solution of the dam-break problem. The outer asymptotic solution can be corrected with the help of the inner solution and matched to this inner solution. In this paper we restrict ourselves to the leading-order inner solution. In order to formulate the matching conditions and to evaluate the dimension of the inner region, the second-order outer solution of the boundary-value problem (2)-(9) is studied in the following section. This second-order outer solution can be potentially used to derive the higher-order inner solution.

\section{Second-order outer solution}

In order to derive the boundary-value problem for the unknown functions $\varphi_{1}(x, y, t), X_{1}(y, t)$ and $Y_{1}(x, t)$, asymptotic expansions of the terms in (2)-(9) close to the initial position of the liquid free surface are used:

$$
\begin{aligned}
& \frac{\partial \varphi}{\partial t}(x, \varepsilon Y(x, t, \varepsilon), t, \varepsilon)=\frac{\partial \varphi_{0}}{\partial t}(x, 0, t)+\varepsilon\left\{\frac{\partial \varphi_{1}}{\partial t}(x, 0, t)+\frac{\partial^{2} \varphi_{0}}{\partial t \partial y}(x, 0, t) Y_{0}(x, t)\right\}+O\left(\varepsilon^{2}\right), \\
& \frac{\partial \varphi}{\partial y}(x, \varepsilon Y(x, t, \varepsilon), t, \varepsilon)=\frac{\partial \varphi_{0}}{\partial y}(x, 0, t)+\varepsilon \frac{\partial \varphi_{1}}{\partial y}(x, 0, t)+O\left(\varepsilon^{2}\right), \\
& \frac{\partial \varphi}{\partial x}(x, \varepsilon Y(x, t, \varepsilon), t, \varepsilon)=\varepsilon\left\{\frac{\partial \varphi_{1}}{\partial x}(x, 0, t)+\frac{\partial^{2} \varphi_{0}}{\partial y \partial x}(x, 0, t) Y_{0}(x, t)\right\}+O\left(\varepsilon^{2}\right), \\
& -p(x, \varepsilon Y(x, t, \varepsilon), t, \varepsilon)=\varepsilon\left\{\frac{\partial \varphi_{1}}{\partial t}(x, 0, t)+\frac{\partial^{2} \varphi_{0}}{\partial y \partial t} Y_{0}(x, t)+\frac{1}{2}\left(\frac{\partial \varphi_{0}}{\partial y}\right)^{2}+Y_{0}(x, t)\right\}+O\left(\varepsilon^{2}\right),
\end{aligned}
$$

where the equality $\varphi_{0}(x, 0, t)=0$ has been taken into account. Similar expansions have been obtained for the part of the free surface, where $x=\varepsilon X(y, t, \varepsilon),-1<y<\varepsilon Y(0, t, \varepsilon)$.

Substituting the obtained asymptotic expansions in (2)-(9) and collecting terms of the order of $O(\varepsilon)$ as $\varepsilon \rightarrow 0$, we arrive at the following boundary-value problem for the second-order velocity potential

$$
\begin{aligned}
& \nabla^{2} \varphi_{1}=0 \quad(x>0,-1<y<0), \\
& \frac{\partial \varphi_{1}}{\partial t}+\frac{\partial^{2} \varphi_{0}}{\partial t \partial y} Y_{0}+\frac{1}{2}\left(\frac{\partial \varphi_{0}}{\partial y}\right)^{2}+Y_{0}=0, \quad \frac{\partial \varphi_{1}}{\partial y}=\frac{\partial Y_{1}}{\partial t} \quad(x>0, y=0), \\
& \frac{\partial \varphi_{1}}{\partial t}+\frac{\partial^{2} \varphi_{0}}{\partial t \partial x} X_{0}+\frac{1}{2}\left[\left(\frac{\partial \varphi_{0}}{\partial y}\right)^{2}+\left(\frac{\partial \varphi_{0}}{\partial x}\right)^{2}\right]=0, \quad \frac{\partial \varphi_{1}}{\partial x}=\frac{\partial \varphi_{0}}{\partial y} \frac{\partial X_{0}}{\partial y}+\frac{\partial X_{1}}{\partial t}(x=0,-1<y<0),
\end{aligned}
$$


$\frac{\partial \varphi_{1}}{\partial y}=0 \quad(x>0, y=-1)$,

$\varphi_{1} \rightarrow 0 \quad(x \rightarrow+\infty)$.

The boundary conditions (29) and (30) can be simplified with the help of new functions $\Phi_{0}(x, y)$ and $\Phi_{1}(x, y)$, where

$\varphi_{0}(x, y, t)=t \Phi_{0}(x, y), \quad \varphi_{1}(x, y, t)=-\frac{1}{3} t^{3} \Phi_{1}(x, y)$,

$Y_{0}(x, t)=\frac{1}{2} t^{2} \frac{\partial \Phi_{0}}{\partial y}(x, 0), \quad X_{0}(y, t)=\frac{1}{2} t^{2} \frac{\partial \Phi_{0}}{\partial x}(0, y)$.

Equations (28)-(33) provide the boundary-value problem for the new unknown function $\Phi_{1}(x, y)$ :

$\nabla^{2} \Phi_{1}=0 \quad(x>0,-1<y<0)$,

$\Phi_{1}=\left(\frac{\partial \Phi_{0}}{\partial y}\right)^{2}+\frac{1}{2} \frac{\partial \Phi_{0}}{\partial y} \quad(x>0, y=0)$,

$\Phi_{1}=\left(\frac{\partial \Phi_{0}}{\partial x}\right)^{2}+\frac{1}{2} \quad(x=0,-1<y<0)$,

$\frac{\partial \Phi_{1}}{\partial y}=0 \quad(x>0, y=-1)$,

$\Phi_{1} \rightarrow 0 \quad(x \rightarrow+\infty)$.

Once the boundary-value problem (34) has been solved, the functions $X_{1}(y, t)$ and $Y_{1}(x, t)$ in (10) are found to be $Y_{1}(x, t)=-\frac{1}{12} t^{4} \frac{\partial \Phi_{1}}{\partial y}(x, 0), \quad X_{1}(y, t)=-\frac{1}{12} t^{4}\left[\frac{\partial \Phi_{1}}{\partial x}(0, y)-\frac{3}{2} \frac{\partial^{2} \Phi_{0}}{\partial x \partial y}\right]$.

Equation (19) gives

$w_{0}(z, t)=t W_{0}(z), \quad W_{0}(z)=\Phi_{0}(x, y)+\mathrm{i} \Psi_{0}(x, y)$.

It can be shown that the function

$\Phi_{10}(x, y)=\mathfrak{R e}\left[\frac{\mathrm{d} W_{0}}{\mathrm{~d} z}\right]^{2}=\left(\frac{\partial \Phi_{0}}{\partial x}\right)^{2}-\left(\frac{\partial \Phi_{0}}{\partial y}\right)^{2}$,

where $\mathfrak{R e}(z)$ denotes the real part of $z$, satisfies Laplace's equation $\nabla^{2} \Phi_{10}=0$ in the flow region $x>0,-1<y<0$ and the boundary conditions

$$
\begin{aligned}
& \Phi_{10}(x, 0)=-\left(\frac{\partial \Phi_{0}}{\partial y}\right)^{2}, \quad \frac{\partial \Phi_{10}}{\partial y}(x,-1)=0 \quad(x>0), \\
& \Phi_{10}(0, y)=\left(\frac{\partial \Phi_{0}}{\partial x}\right)^{2}-1 \quad(-1<y<0) .
\end{aligned}
$$

It is advantageous to decompose the unknown potential $\Phi_{1}(x, y)$ as

$\Phi_{1}(x, y)=\Phi_{10}(x, y)+\tilde{\Phi}_{1}(x, y)$,

where the new unknown function $\tilde{\Phi}_{1}(x, y)$ satisfies equations

$\nabla^{2} \tilde{\Phi}_{1}=0 \quad(x>0,-1<y<0)$,

$\tilde{\Phi}_{1}=2\left(\frac{\partial \Phi_{0}}{\partial y}\right)^{2}+\frac{1}{2} \frac{\partial \Phi_{0}}{\partial y} \quad(x>0, y=0)$, 
$\tilde{\Phi}_{1}=\frac{3}{2} \quad(x=0,-1<y<0)$,

$\frac{\partial \tilde{\Phi}_{1}}{\partial y}=0 \quad(x>0, y=-1)$,

$\tilde{\Phi}_{1} \rightarrow 0 \quad(x \rightarrow+\infty)$.

In (41), the derivative $\left(\partial \Phi_{0} / \partial y\right)(x, 0)$ is equal to

$\frac{\partial \Phi_{0}}{\partial y}(x, 0)=-\frac{4}{\pi} \arctan [\exp (-\pi x / 2)]$

and $\left(\partial \Phi_{0} / \partial y\right)(x, 0) \rightarrow-1$ as $x \rightarrow+0$. Correspondingly, $\tilde{\Phi}_{1}(x, 0) \rightarrow \frac{3}{2}$ as $x \rightarrow+0$, which implies that the function $\tilde{\Phi}_{1}$ is continuous along the liquid free surface.

The function $\tilde{\Phi}_{1}(x, y)$ is continued symmetrically with respect to the bed, $y=-1$, into the region $-2<y<-1$, $x>0$. After the continuation one needs to find the potential $\tilde{\Phi}_{1}(x, y)$, which satisfies Laplace's equation in the region $-2<y<0, x>0$, is equal to $\frac{3}{2}$ on $-2<y<-1, x=0$ and is given by (41) and (45) where $x>0, y=-2$ and $y=0$. This new boundary-value problem is solved by using the complex transformation

$\zeta_{1}=-\cosh z_{1}, \quad z_{1}=\frac{\pi}{2}[x+\mathrm{i}(y+2)], \quad \zeta_{1}=\xi_{1}+\mathrm{i} \eta_{1}$,

which maps the region $-2<y<0, x>0$ onto the lower half-plane $\eta_{1}<0$ (see Fig. 2). The interval $-2<y<-1$, $x=0$ corresponds to the interval $\eta_{1}=0,-1<\xi_{1}<1$, where $\xi_{1}=\cos (\pi y / 2)$. The upper free surface, $x>0, y=0$, corresponds to the interval $\eta_{1}=0, \xi_{1}>1$, where $\xi_{1}=\cosh (\pi x / 2)$. The line, $x>0, y=-2$, corresponds to the interval $\eta_{1}=0, \xi_{1}<-1$, where $\xi_{1}=-\cosh (\pi x / 2)$. The bottom line, $x>0, y=-1$, corresponds to the line $\xi_{1}=0, \eta_{1}=-\sinh (\pi x / 2)$. For $x>0$ and $y=0$ we find $\mathrm{e}^{-\frac{\pi x}{2}}=\xi_{1}-\sqrt{\xi_{1}^{2}-1}$, which provides the boundary conditions for the harmonic function $\bar{\Phi}_{1}\left(\xi_{1}, \eta_{1}\right)=\tilde{\Phi}_{1}\left(x\left(\xi_{1}, \eta_{1}\right), y\left(\xi_{1}, \eta_{1}\right)\right)$ in the form

$$
\begin{aligned}
& \bar{\Phi}_{1}\left(\xi_{1}, 0\right)=\frac{32}{\pi^{2}} \arctan ^{2}\left[\left|\xi_{1}\right|-\sqrt{\xi_{1}^{2}-1}\right]-\frac{2}{\pi} \arctan \left[\left|\xi_{1}\right|-\sqrt{\xi_{1}^{2}-1}\right] \quad\left(\left|\xi_{1}\right|>1\right), \\
& \bar{\Phi}_{1}\left(\xi_{1}, 0\right)=\frac{3}{2} \quad\left(-1<\xi_{1}<1\right) .
\end{aligned}
$$

Note that the potential $\bar{\Phi}_{1}\left(\xi_{1}, 0\right)$ is continuous along the boundary $\eta_{1}=0$ but its derivative $\left(\partial \bar{\Phi}_{1} / \partial \xi_{1}\right)\left(\xi_{1}, 0\right)$ is singular at the points $\xi_{1}= \pm 1$ :

$$
\begin{aligned}
& \frac{\partial \bar{\Phi}_{1}}{\partial \xi_{1}}\left(\xi_{1}, 0\right)=\frac{1}{\pi \xi_{1} \sqrt{\xi_{1}^{2}-1}}\left(1-\frac{32}{\pi} \arctan \left[\left|\xi_{1}\right|-\sqrt{\xi_{1}^{2}-1}\right]\right) \quad\left(\left|\xi_{1}\right|>1\right), \\
& \frac{\partial \bar{\Phi}_{1}}{\partial \xi_{1}}\left(\xi_{1}, 0\right)=0 \quad\left(\left|\xi_{1}\right|<1\right) .
\end{aligned}
$$

It is known (see [17, p. 83]) that

$$
\frac{\partial \bar{\Phi}_{1}}{\partial \eta_{1}}\left(\xi_{1}, 0\right)=-\frac{1}{\pi} \int_{-\infty}^{\infty} \frac{\partial \bar{\Phi}_{1}}{\partial \xi_{1}}(\tau, 0) \frac{\mathrm{d} \tau}{\tau-\xi_{1}}
$$

for a function $\bar{\Phi}_{1}\left(\xi_{1}, \eta_{1}\right)$ which is analytical in the lower half-plane. In our case (48) and (49) give

$$
\frac{\partial \bar{\Phi}_{1}}{\partial \eta_{1}}\left(\xi_{1}, 0\right)=\frac{2}{\pi^{2}} \int_{1}^{\infty} \frac{\frac{32}{\pi} \arctan \left[\tau-\sqrt{\tau^{2}-1}\right]-1}{\sqrt{\tau^{2}-1}\left(\tau^{2}-\xi_{1}^{2}\right)} \mathrm{d} \tau \quad\left(-\infty<\xi_{1}<\infty\right) .
$$

It is convenient to introduce a new integration variable in (50) as $u=\tau-\sqrt{\tau^{2}-1}$; then

$$
\frac{\partial \bar{\Phi}_{1}}{\partial \eta_{1}}\left(\xi_{1}, 0\right)=\frac{8}{\pi^{2}} \int_{0}^{1}\left[\frac{32}{\pi} \arctan u-1\right] \frac{u \mathrm{~d} u}{\left(u^{2}+1-2 u\left|\xi_{1}\right|\right)\left(u^{2}+1+2 u\left|\xi_{1}\right|\right)} .
$$


Fig. 2 Conformal mapping of the extended flow region (above) onto lower half-plane (below)
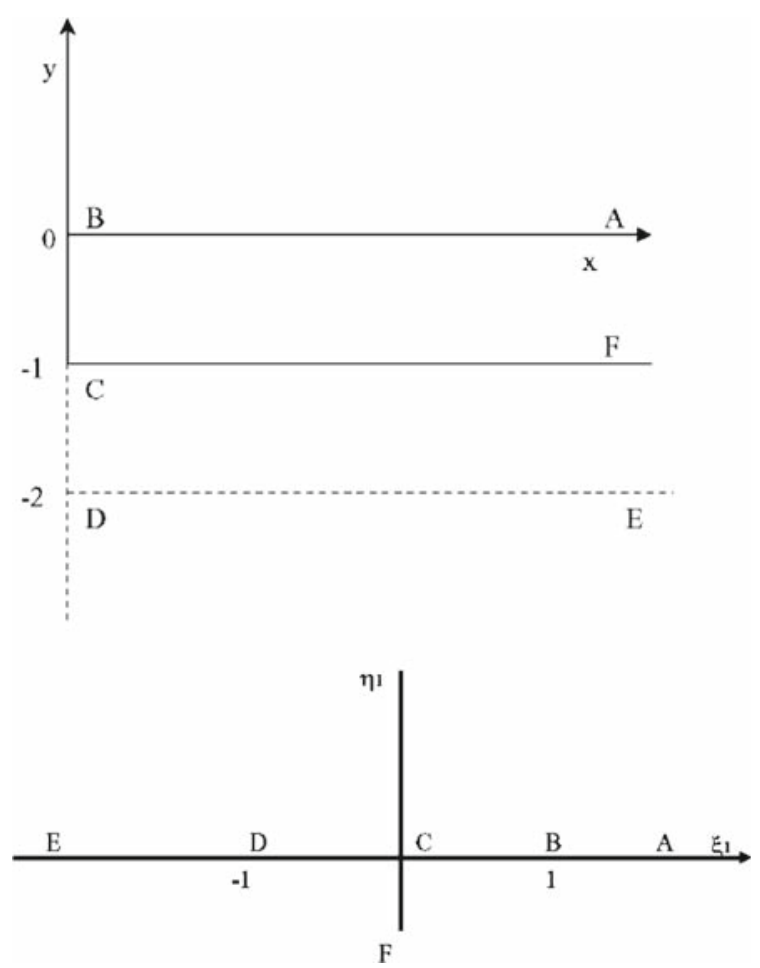

The integral in (51) is singular, when $\left|\xi_{1}\right| \geq 1$, and regular, when $\left|\xi_{1}\right|<1$. Once the integral (51) has been evaluated, the normal derivatives of the potential $\tilde{\Phi}_{1}(x, y)$ on the free surface are given as

$$
\begin{aligned}
& \frac{\partial \tilde{\Phi}_{1}}{\partial x}(0, y)=\frac{\pi}{2} \sin \left(\frac{\pi y}{2}\right) \frac{\partial \bar{\Phi}_{1}}{\partial \eta_{1}}\left[\cos \left(\frac{\pi y}{2}\right), 0\right] \quad(-1<y<0), \\
& \frac{\partial \tilde{\Phi}_{1}}{\partial y}(x, 0)=\frac{\pi}{2} \sinh \left(\frac{\pi x}{2}\right) \frac{\partial \bar{\Phi}_{1}}{\partial \eta_{1}}\left[\cosh \left(\frac{\pi x}{2}\right), 0\right] \quad(x>0) .
\end{aligned}
$$

These derivatives are needed to compute the functions $X_{1}(y, t)$ and $Y_{1}(x, t)$ in (35) and to reconstruct the secondorder deflection of the liquid free surface outside a small neighbourhood of the intersection point. The first-order and the second-order deflections of the free surface $x=\varepsilon X(y, t, \varepsilon)$ are shown in Fig. 3 where $\varepsilon=0.1$ and $t=1$.

Special care is required to calculate the free-surface shape near the point $x=0, y=\varepsilon Y(0, t, \varepsilon)$, where the horizontal and vertical free surface join up. Let us show that the obtained second-order outer solution provides $X(\varepsilon Y(0, t, \varepsilon), t, \varepsilon)=O\left(\varepsilon^{2}\right)$ as $\varepsilon \rightarrow 0$. The third equation in (10) gives

$$
\begin{aligned}
X(\varepsilon Y(0, t, \varepsilon), t, \varepsilon) & =X_{0}(\varepsilon Y(0, t, \varepsilon), t)+\varepsilon X_{1}(\varepsilon Y(0, t, \varepsilon), t)+O\left(\varepsilon^{2}\right) \\
& =X_{0}(0, t)+\varepsilon\left[X_{1}(0, t)+\frac{\partial X_{0}}{\partial y}(0, t) Y_{0}(0, t)\right]+O\left(\varepsilon^{2}\right) .
\end{aligned}
$$

Equations (16), (17) and (35) yield

$$
X_{0}(0, t)=0, \quad \frac{\partial X_{0}}{\partial y}(0, t)=\frac{1}{2} t^{2}, \quad Y_{0}(0, t)=-\frac{1}{2} t^{2}, \quad X_{1}(0, t)=-\frac{1}{12} t^{4}\left[\frac{\partial \Phi_{1}}{\partial x}(0,0)-\frac{3}{2} \frac{\partial^{2} \Phi_{0}}{\partial x \partial y}(0,0)\right] .
$$

Here

$$
\frac{\partial \Phi_{1}}{\partial x}(0,0)=2 \frac{\partial \Phi_{0}}{\partial y}(0,0) \frac{\partial^{2} \Phi_{0}}{\partial x \partial y}(0,0)+\frac{1}{2} \frac{\partial^{2} \Phi_{0}}{\partial x \partial y}(0,0),
$$




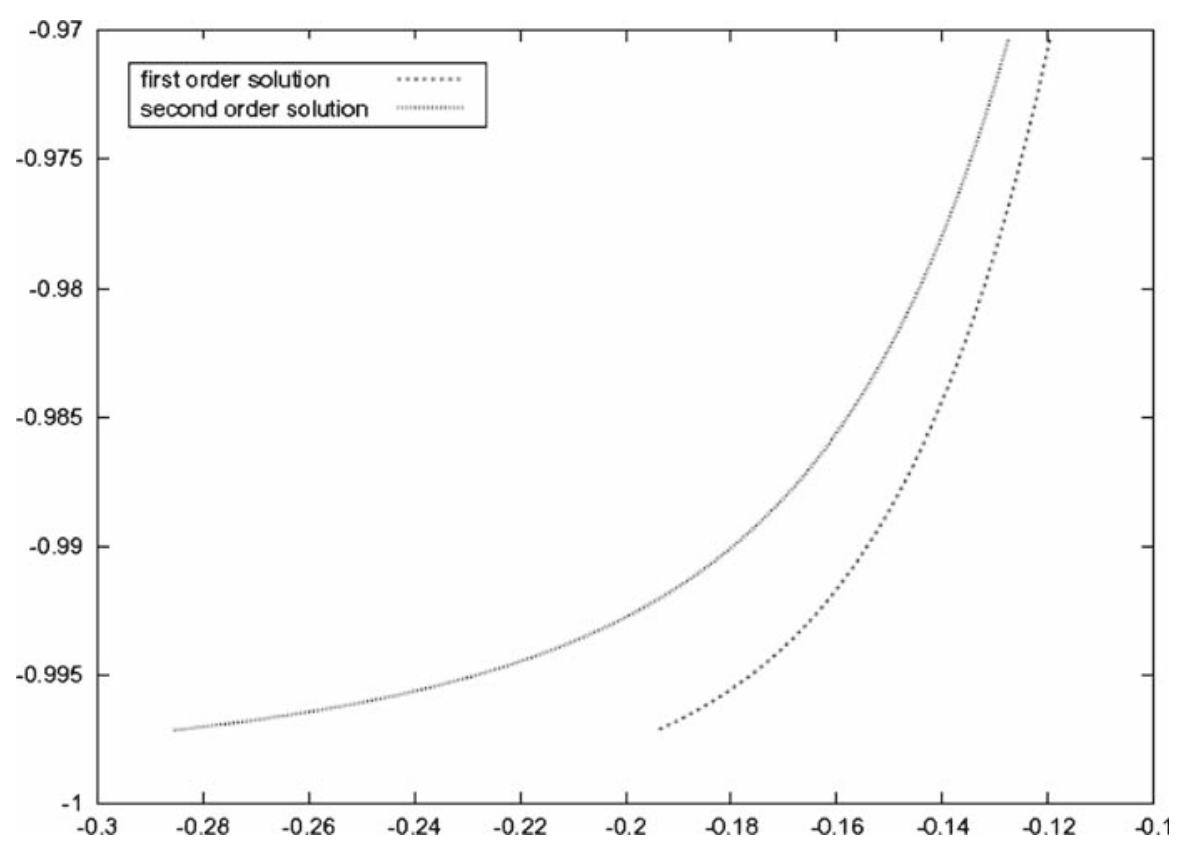

Fig. 3 The non-dimensional shape of the liquid free surface close to the bottom: the first-order outer solution is shown by dashed line and the second-order outer solution by solid line for $\varepsilon=0.1$ and $t=1$

$\frac{\partial^{2} \Phi_{0}}{\partial x \partial y}(0,0)=1, \quad \frac{\partial \Phi_{0}}{\partial y}(0,0)=-1$,

which follow from the boundary conditions in (34), (11) and (33). We obtain

$\frac{\partial \Phi_{1}}{\partial x}(0,0)=-\frac{3}{2}, \quad X_{1}(0, t)=\frac{1}{4} t^{4}$

and finally

$X_{1}(0, t)+\frac{\partial X_{0}}{\partial y}(0, t) Y_{0}(0, t)=0$.

Therefore, the original equation $X(\varepsilon Y(0, t, \varepsilon), t, \varepsilon)=0$ is satisfied with the obtained second-order solution up to the terms of the order of $O\left(\varepsilon^{2}\right)$ as $\varepsilon \rightarrow 0$.

We conclude that close to the intersection point $\tilde{\Phi}_{1}(x, y)=O(1)$ as $x \rightarrow 0$ and $y \rightarrow-1$. Therefore, the singular behavior of the potential $\Phi_{1}(x, y)$ is described by the function $\Phi_{10}(x, y)$ which is given by (37). Within the inner variables $x=a \xi, y=-1+a \eta$

$\varphi_{1}=-\frac{1}{3} t^{3} \mathfrak{R e}\left(\frac{\mathrm{d} W_{0}}{\mathrm{~d} z}\right)^{2}+O(1) \quad(a \rightarrow 0)$.

The asymptotic formula (21) gives us

$\varphi_{1}=-\frac{1}{3} t^{3} \cdot \frac{4}{\pi^{2}} \mathfrak{R e}\left(\log ^{2}\left[\frac{\pi}{4} a \zeta\right]\right)+O(1)$

and

$\varphi_{1}=-\frac{4}{3 \pi^{2}} t^{3}\left(\log ^{2} a+2 \log \frac{\pi}{4} \cdot \log a+\log a \log \left(\xi^{2}+\eta^{2}\right)\right)+O(1)$. 
Equations (10), (23) and (55) give the asymptotic behavior of the second-order outer solution close to the intersection point in the form

$$
\begin{aligned}
\varphi(x, y, t)= & t+a \log a \cdot \frac{2 t}{\pi} \xi+a \cdot \frac{2 t}{\pi}\left\{\frac{\xi}{2} \log \left[\xi^{2}+\eta^{2}\right]+\xi\left(\log \frac{\pi}{4}-1\right)-\eta \arctan \left(\frac{\eta}{\xi}\right)\right\} \\
& -\varepsilon \log ^{2} a \cdot \frac{4 t^{3}}{3 \pi^{2}}-\varepsilon \log a \cdot \frac{4 t^{3}}{3 \pi^{2}}\left\{2 \log \frac{\pi}{4}+\log \left[\xi^{2}+\eta^{2}\right]\right\}+O\left(a^{3}\right)+O(\varepsilon) .
\end{aligned}
$$

Equations (23) and (55) show that, close to the intersection point, the first- and second-order terms in the asymptotic expansion (10) are of the same order if and only if

$a \log a=-\varepsilon \log ^{2} a$.

Hence the size of the inner region, where the outer solution fails, is given in implicit form as

$a=-\varepsilon \log a$.

In the following we consider $a(\varepsilon)$ as the new small parameter and $\varepsilon=-a / \log a$, where $\varepsilon=o(a)$ as $a \rightarrow 0$. Then the asymptotic formula (56) takes the form

$$
\begin{aligned}
\varphi(x, y, t)= & t+a \log a\left\{\frac{2 t}{\pi} \xi+\frac{4 t^{3}}{3 \pi^{2}}\right\} \\
& +a\left\{\frac{2 t}{\pi}[\xi \log \rho-\eta \theta]+\xi\left(\log \frac{\pi}{4}-1\right)+\frac{8 t^{3}}{3 \pi^{2}} \log \left(\frac{\pi \rho}{4}\right)\right\}+O\left(\frac{a}{\log a}\right),
\end{aligned}
$$

where $\xi=\rho \cos \theta$ and $\eta=\rho \sin \theta$. More terms in (58) can be obtained if one considers the asymptotic expansion of the potential $\tilde{\Phi}_{1}(x, y)$ close to the intersection point.

In the inner variables $\xi$ and $\eta$ the second-order outer asymptotics of the free-surface shape close to the bottom give us

$\xi=-\frac{t^{2}}{\pi}-\frac{1}{\log a} \cdot \frac{t^{2}}{\pi} \log \left(\frac{\pi \eta}{4}\right)-\frac{1}{\log ^{2} a} \cdot \frac{t^{4}}{12 \pi} \frac{1}{\eta}+O\left(\frac{a^{2}}{\log a}\right)$

as $a \rightarrow 0$.

Asymptotic formulae (58) and (59) are used to formulate the far-field conditions for the potential and the free-surface shape in the inner region.

\section{Inner asymptotics of the flow close to the intersection point}

The asymptotic formulae (58) and (59) suggest that the inner velocity potential $\varphi_{i}(\xi, \eta, t, \varepsilon)$ and the free-surface shape close to the bottom can be found as follows:

$$
\begin{aligned}
& \varphi_{i}(\xi, \eta, t, \varepsilon)=t+a \log a\left\{\frac{2 t}{\pi} \xi+\frac{4 t^{3}}{3 \pi^{2}}\right\}+a \Phi_{i}(\xi, \eta, t)+O\left(\frac{a}{\log a}\right), \\
& \xi=-\frac{t^{2}}{\pi}-\frac{1}{\log a} \mathcal{H}(\eta, t)+O\left(\frac{1}{\log ^{2} a}\right) .
\end{aligned}
$$

The function $X(y, t, \varepsilon)$, which describes the shape of the free surface in the kinematic boundary condition (5), is now given as

$$
X(y, t, \varepsilon)=\frac{t^{2}}{\pi} \log a+\mathcal{H}(\eta, t)+O\left(\frac{1}{\log a}\right) .
$$

Substituting (62) and (60) in equations (2), (3), (5)-(8), we obtain the pressure distribution in the inner region

$$
-p=a \log a\left\{\frac{2}{\pi} \xi+\frac{2 t^{2}}{\pi^{2}}\right\}+a\left\{\frac{\partial \Phi_{i}}{\partial t}-\frac{2 t}{\pi} \frac{\partial \Phi_{i}}{\partial \xi}+\eta\right\}+O\left(\frac{a}{\log a}\right)
$$


the kinematic condition (5) gives

$\frac{\partial \Phi_{i}}{\partial \xi}=\frac{\partial \mathcal{H}}{\partial t}+O\left(\frac{1}{\log a}\right)$

the dynamic boundary condition on the free surface (61), taking into account (63), gives

$\frac{\partial \Phi_{i}}{\partial t}-\frac{2 t}{\pi} \frac{\partial \Phi_{i}}{\partial \xi}-\frac{2}{\pi} \mathcal{H}(\eta, t)+\eta=O\left(\frac{1}{\log a}\right)$.

The boundary conditions (64) and (65) must be satisfied on the free surface (61), where $1 / \log a \rightarrow 0$ as $a \rightarrow 0$. Therefore, these conditions can be imposed at leading order on the vertical moving line $\xi=-t^{2} / \pi$.

The function $\Phi_{i}(\xi, \eta, t)$ is harmonic in the flow region $\xi>-t^{2} / \pi$ and satisfies the condition $\partial \Phi_{i} / \partial \eta=0$ on the bottom $\eta=0$. Moreover, the unknown functions $\Phi_{i}(\xi, \eta, t)$ and $\mathcal{H}(\eta, t)$ have to be matched with the outer solution defined by (58) and (59) in the far field. The matching conditions at leading order are

$\Phi_{i}(\xi, \eta, t)=\Phi_{i}^{\infty}(\xi, \eta, t)+o(1), \quad \mathcal{H}(\eta, t)=\frac{t^{2}}{\pi} \log \left(\frac{\pi \eta}{4}\right)+o(1) \quad(\rho \rightarrow \infty)$,

where

$\Phi_{i}^{\infty}(\xi, \eta, t)=\frac{2 t}{\pi}[\xi \log \rho-\eta \theta]+\xi\left(\log \frac{\pi}{4}-1\right)+\frac{8 t^{3}}{3 \pi^{2}} \log \left(\frac{\pi \rho}{4}\right)$,

which follows from (58). Here the function $\Phi_{i}^{\infty}(\xi, \eta, t)$ as given in (67) is harmonic and satisfies the bottom boundary condition, but it is singular at $\xi=0, \eta=0$, which is a point inside the flow domain.

It is convenient to introduce new variables $x_{1}$ and $y_{1}$ as

$\xi=-\frac{t^{2}}{\pi}-y_{1}, \quad \eta=x_{1}$

and continue the new unknown functions $\phi\left(x_{1}, y_{1}, t\right)=\Phi_{i}\left(-t^{2} / \pi-y_{1}, x_{1}, t\right)$ and $h\left(x_{1}, t\right)=-\mathcal{H}\left(x_{1}, t\right)$ symmetrically with respect to the bottom line $x_{1}=0$. Conditions (64) and (65) provide at leading order

$\frac{\partial \phi}{\partial t}+\frac{2}{\pi} h+\left|x_{1}\right|=0, \quad \frac{\partial \phi}{\partial y_{1}}=\frac{\partial h}{\partial t} \quad\left(y_{1}=0,-\infty<x_{1}<\infty\right)$,

which gives the boundary condition

$\frac{\partial^{2} \phi}{\partial t^{2}}+\frac{2}{\pi} \frac{\partial \phi}{\partial y_{1}}=0 \quad\left(y_{1}=0,-\infty<x_{1}<\infty\right)$

and initial conditions

$\frac{\partial \phi}{\partial t}\left(x_{1}, 0,0\right)=-\left|x_{1}\right|, \quad \phi\left(x_{1}, 0,0\right)=0$.

The potential $\phi\left(x_{1}, y_{1}, t\right)$ satisfies Laplace's equation

$\nabla^{2} \phi=0 \quad\left(y_{1}<0\right)$

and the far-field condition from Eq. 66

$\phi\left(x_{1}, y_{1}, t\right)=\Phi_{i}^{\infty}\left(-t^{2} / \pi-y_{1}, x_{1}, t\right)+o(1) \quad(\rho \rightarrow \infty)$.

The boundary condition (69) for Laplace's equation (71) is a feature of Cauchy-Poisson problem [2] for water waves that are generated by initial disturbances of the free surface (70) and a "generator" in the far field (72). The non-trivial far-field condition (72) makes the Cauchy-Poisson problem (69)-(72) difficult to solve. In order to resolve this difficulty, we seek a solution of the form

$\phi\left(x_{1}, y_{1}, t\right)=\phi^{\infty}\left(x_{1}, y_{1}, t\right)+\psi\left(x_{1}, y_{1}, t\right)$,

where $\phi^{\infty}\left(x_{1}, y_{1}, t\right)$ satisfies the far-field condition (72), Laplace's equation (71) and is regular in the region $y_{1}<0$. Note that the function $\Phi_{i}^{\infty}\left(-t^{2} / \pi-y_{1}, x_{1}, t\right)$ in (72) satisfies the far-field condition (72) and Laplace's equation (71) but is singular at the point $x_{1}=0, y_{1}=-t^{2} / \pi$, which is inside the flow region $y_{1}<0$. 
In order to construct the function $\phi^{\infty}\left(x_{1}, y_{1}, t\right)$, we introduce a polar coordinate system $(r, \bar{\theta})$, with its origin being outside the flow region

$x_{1}=r \cos \bar{\theta}, \quad y_{1}=\alpha t^{2}+r \sin \bar{\theta}$.

Here $\alpha$ is a positive parameter, the value of which will be specified later. Substituting (74) in the function $\Phi_{i}^{\infty}\left(-t^{2} / \pi-y_{1}, x_{1}, t\right)$ and letting $r \rightarrow \infty$, we obtain

$\Phi_{i}^{\infty}\left(-t^{2} / \pi-\alpha t^{2}-r \sin \bar{\theta}, r \cos \bar{\theta}, t\right)=\phi^{\infty}\left(x_{1}, y_{1}, t\right)+O(1 / r)$,

where

$\phi^{\infty}\left(x_{1}, y_{1}, t\right)=\frac{2 t}{\pi}\left\{-y_{1} \log \left(\frac{\pi r}{4}\right)-x_{1}\left(\frac{\pi}{2}+\bar{\theta}\right)+\frac{t^{2}}{3 \pi} \log \left(\frac{\pi r}{4}\right)-\alpha t^{2}+y_{1}\right\}, \quad\left(0<x_{1}<\infty\right)$.

The function (75) satisfies the far-field condition (72), Laplace's equation (71) and is regular in the flow region $y_{1}<0$.

The unknown function $h\left(x_{1}, t\right)$ is sought in the form

$h\left(x_{1}, t\right)=-\frac{t^{2}}{\pi} \log \left(\frac{\pi}{4} \sqrt{x_{1}^{2}+\alpha^{2} t^{4}}\right)+\bar{h}\left(x_{1}, t\right)$.

The far-field conditions (66) imply

$\psi\left(x_{1}, y_{1}, t\right) \rightarrow 0, \quad \bar{h}\left(x_{1}, t\right) \rightarrow 0 \quad\left(\left|x_{1}\right| \rightarrow \infty, \quad y_{1} \rightarrow-\infty\right)$.

Substituting (73) and (76) in (68) and (69), we find

$$
\begin{aligned}
& \frac{\partial \psi}{\partial t}+\frac{2}{\pi} \bar{h}=\frac{2}{\pi}\left(x_{1} \bar{\theta}+\alpha t^{2}\right)+\frac{4 \alpha^{2} t^{6}}{\pi r^{2}}\left(\alpha-\frac{1}{3 \pi}\right)+x_{1}-\left|x_{1}\right|, \quad\left(y_{1}=0,-\infty<x_{1}<\infty\right), \\
& \frac{\partial \bar{h}}{\partial t}-\frac{\partial \psi}{\partial y_{1}}=\frac{2 \alpha}{\pi}\left(2 \alpha-\frac{1}{3 \pi}\right) \frac{t^{5}}{x_{1}^{2}+\alpha^{2} t^{4}}, \quad\left(y_{1}=0,-\infty<x_{1}<\infty\right),
\end{aligned}
$$

which gives the boundary condition

$\frac{\partial^{2} \psi}{\partial t^{2}}+\frac{2}{\pi} \frac{\partial \psi}{\partial y_{1}}=-\frac{8}{3 \pi^{2}} \frac{t^{5}}{t^{4}+9 \pi^{2} x_{1}^{2}}, \quad\left(y_{1}=0,-\infty<x_{1}<\infty\right)$,

where $\alpha=\frac{1}{3 \pi}$ was used to simplify the calculations.

We obtained that the potential $\psi\left(x_{1}, y_{1}, t\right)$ satisfies the following equations

$\nabla^{2} \psi=0 \quad\left(y_{1}<0\right)$,

$\frac{\partial^{2} \psi}{\partial t^{2}}+\frac{2}{\pi} \frac{\partial \psi}{\partial y_{1}}=-\frac{8}{3 \pi^{2}} \frac{t^{5}}{t^{4}+9 \pi^{2} x_{1}^{2}}, \quad\left(y_{1}=0,-\infty<x_{1}<\infty\right)$,

$\psi\left(x_{1}, 0,0\right)=0, \quad \frac{\partial \psi}{\partial t}\left(x_{1}, 0,0\right)=0$,

$\psi \rightarrow 0 \quad\left(x_{1}^{2}+y_{1}^{2} \rightarrow \infty\right)$.

Once the potential $\psi\left(x_{1}, y_{1}, t\right)$ has been computed as the solution of the Cauchy-Poisson problem (80), the function $h\left(x_{1}, t\right)$, which describes the shape of the free surface close to the bottom, is obtained from (78) as

$h\left(x_{1}, t\right)=x_{1} \bar{\theta}+\frac{1}{3 \pi} t^{2}-\frac{\pi}{2} \frac{\partial \psi}{\partial t}\left(x_{1}, 0, t\right)$.

The boundary-value problem (80) is solved with the help of a Fourier transform in $x_{1}$. The time derivative of the potential in (81) can be obtained in the form

$\frac{\partial \psi}{\partial t}\left(x_{1}, 0, t\right)=-t^{2} S(u), \quad u=\frac{x_{1}}{t^{2}}$, 
(a)

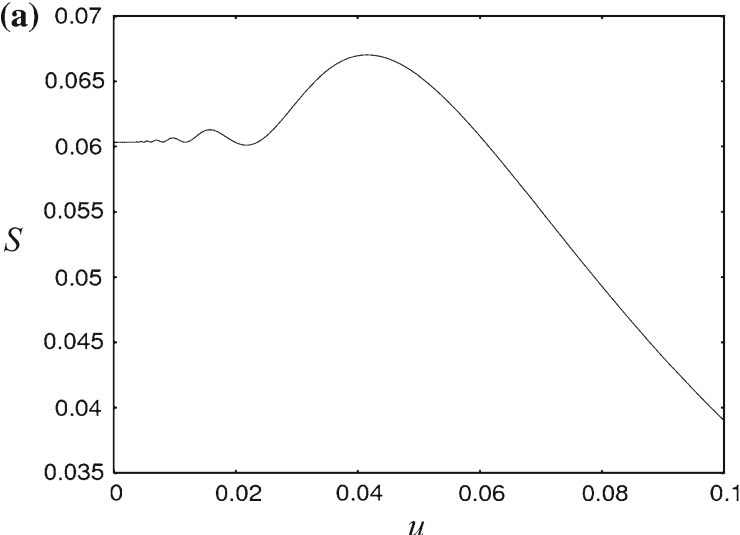

(b)

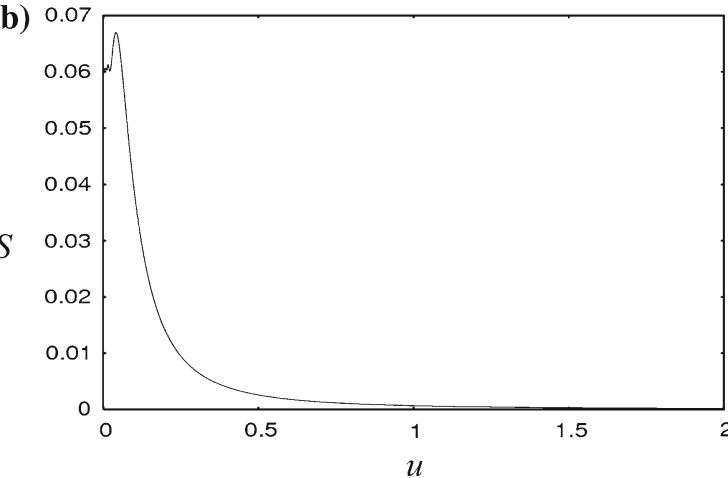

Fig. 4 Function $S(u)$
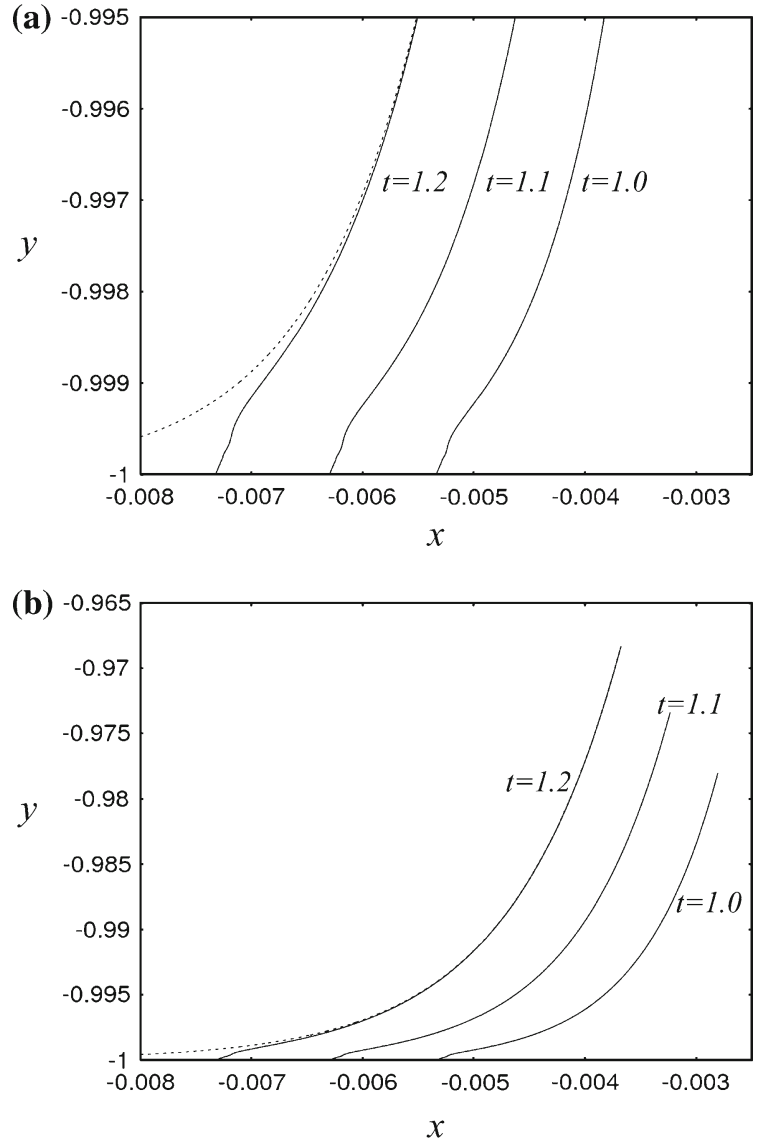

Fig. 5 Positions of the free surface predicted by the leading-order inner solution close to the bed at $t=1.0,1.1,1.2$ for $\varepsilon=0.002$. The leading-order outer solution is shown by dotted line at $t=1.2$

$S(u)=\frac{8}{9 \pi^{2}} \int_{0}^{\infty}\left(\left(3-\tau^{2}\right) \exp \left(-\frac{1}{6} \tau^{2}\right)-3 \cos \tau\right) \cos \left[\frac{\pi}{2} \tau^{2} u\right] \frac{\mathrm{d} \tau}{\tau^{3}}$,

where $S(0) \approx 0.06$ and $S^{\prime}(0)=0$. The function $S(u)$ is depicted in Fig. 4. The function exhibits high-frequency oscillations for small $u$; this is, close to the bed.

Equations (61) and (81)-(83) provide the shape of the inner free surface as

$x=\frac{1}{\pi} \varepsilon t^{2} \log \left(a(\varepsilon) t^{2}\right)+\varepsilon t^{2}\left\{\frac{1}{\pi} \log \left(\frac{\pi}{4} \sqrt{u^{2}+\frac{1}{9 \pi^{2}}}\right)+u \arctan \left(\frac{1}{3 \pi u}\right)-\frac{1}{3 \pi}-\frac{\pi}{2} S(u)\right\}$,

where $u=(y+1) /\left(a(\varepsilon) t^{2}\right)$ and $a(\varepsilon)$ is the solution of the equation $a=-\varepsilon \log a$. It is seen that the free-surface shape predicted by the leading-order inner solution is self-similar in the coordinate system moving along the bed as $x_{0}(t)=\pi^{-1} \varepsilon t^{2} \log \left[a t^{2}\right]$. The self-similar variables are $\left(x-x_{0}(t)\right) /\left(a t^{2}\right)$ and $(y+1) /\left(a t^{2}\right)$.

The free-surface shapes predicted by the leading-order inner solution (84) close to the bottom are shown in Fig. 5 for $\varepsilon=0.002$ and different time instants, $t=1, t=1.1, t=1.2$ in non-dimensional variables (1). The dotted line represents the leading-order outer solution at $t=1.2$. It is seen that the outer and inner solutions match each other well. The inner solution describes a jet which propagates along the dry bed (see Fig. 5b). At leading order the dimensional horizontal coordinate $x_{j}^{\prime}\left(t^{\prime}\right)$ of the jet head is estimated as 
$x_{j}^{\prime}\left(t^{\prime}\right) \approx \frac{1}{\pi} g t^{\prime 2} \log \left(\frac{g t^{\prime 2}}{H}\right)$.

This asymptotic formula corresponds to that derived by King and Needham [4] in the problem of the flow generated by a uniformly accelerated vertical plate if the plate acceleration is equal to the gravitational acceleration $g$ (see asymptotic formula (3.1) in [4]). Figure 5a and numerical analysis demonstrate that the angle between the free surface and the bed decreases with time. Equation (84) and asymptotic formulae: $t=O(1), \log a \sim \log \varepsilon$ as $\varepsilon \rightarrow 0$, show that in the leading order

$\frac{\mathrm{d} y^{\prime}}{\mathrm{d} x^{\prime}} \sim-\frac{2}{\pi} \log \left(\frac{g t^{\prime 2}}{H}\right)$

at the intersection point. The latter formula coincides with that derived by King and Needham (see asymptotic formula (2.29) in [4]). It is interesting to note that in the problem of a uniformly accelerated vertical plate considered in [4], the angle between the free surface and the moving plate is independent of the plate acceleration in the leading order for small time.

\section{Concluding remarks}

We derived the leading-order asymptotic solution of the two-dimensional dam-break problem. It has been shown that the flow close to the intersection point is self-similar and the angle between the free surface and the bottom decreases in time. The free-surface shape close to the bottom exhibits oscillations, the amplitude of which decays in the far-field and when approaching the bed.

In this study we restricted ourselves to the leading-order inner solution. However, the higher-order inner solution can be derived in principle and matched to the second-order outer solution from Sect. 3.

The present analysis of dam-break flow is similar to the asymptotic analysis of the flow generated by a uniformly accelerating plate as performed by King and Needham [4]. The similarity of these two problems becomes clear if in the problem studied in [4] we neglect gravity, assume that the plate acceleration is equal to the gravitational acceleration $g$ and formulate the problem in the coordinate system moving together with the plate. The configurations of the flows will still be different but this difference is expected to affect only the outer solution and some coefficients in the matching conditions but not the structure of these conditions. This similarity and the experience gained from [4] made it possible, in particular, to avoid formulating the nonlinear boundary-value problem for the leading-order inner solution (see (2.20) and (2.21) in [4]) and to seek the leading-order inner solution in the form given by (60) and (61).

King and Needham [4] did not use the second-order outer solution to determine the size of the inner region and the matching conditions. We derived the second-order outer solution and used this to obtain the matching conditions. This procedure is similar to that presented in [16], where the initial flow generated by an impulsively moved plate was studied. Comparing [4] and [16], it is possible for us to understand why the second-order outer solution is not required in the asymptotic analysis of [4] but is needed in [16]. Note that in the present analysis the second-order outer solution gives a contribution to the matching condition for the velocity potential but not to the matching condition for the free-surface shape (see (66) and (67)). This is why, even though the second-order outer solution participates in the matching condition, the leading-order inner solution predicts the free-surface shape close to the bed, which matches the free-surface shape provided by the leading-order outer solution but not by the second-order outer solution.

In our problem the second-order outer solution is represented in the matching conditions by the second and the third terms in (67). We cannot solve the inner boundary-value problem without these terms because they do not decay in the far field of the inner region. A similar situation took place in the analysis by King and Needham (see [4, Appendix A], the paragraph with equation (A14)). They did not consider the second-order outer solution and their matching condition for the velocity potential contained only one term similar to the first term in (67). However, they needed additional terms in their matching to solve the leading-order inner problem and explained a reason 
for that in the Appendix. They recovered these terms by using the asymptotic analysis of the solution of the inner boundary-value problem in the far field (see [4, Eq. A14]). This means that the proper matching conditions can be formulated by using only the leading-order outer solution but a further analysis of the inner solution in the far field is required. In our problem the suitable matching conditions were obtained by using the second-order outer solution, which can be also used to derive the higher-order inner solution. In addition, the matching conditions used in the present paper make it possible to formulate the inner boundary-value problem in rather simple form (80) and to find the inner solution (83) and (84) which is easy to analyse.

Acknowledgements This work was carried out while the first author was visiting the Izmir Institute of Technology in Turkey. The authors are grateful to the Scientific and Technological Research Council of Turkey and the International Centre for Theoretical Physics-Trieste for supporting this visit. The authors are also grateful to the referees, who suggested several important improvements to the presentation of the results, and to Dr. Mark Cooker from University of East Anglia who read the paper and commented on it.

\section{References}

1. Pohle FV (1950) The Lagrangian equations of hydrodynamics: solutions which are analytic functions of time. PhD dissertation, New York University, USA

2. Stoker JJ (1957) Water waves. Interscience Publishers Inc, New York

3. Korobkin AA, Pukhnachov VV (1988) Initial stage of water impact. Ann Rev Fluid Mech 20:159-185

4. King AC, Needham DJ (1994) The initial development of a jet caused by fluid, body and free surface interaction. Part 1 . A uniformly accelerating plate. J Fluid Mech 268:89-101

5. Stansby PK, Chegini A, Barnes TCD (1998) The initial stages of dam-break flow. J Fluid Mech 374:407-424

6. Zoppou C, Roberts S (2003) Explicit schemes for dam-break simulations. J Hydraul Eng 129:11-34

7. Glaister P (1991) Solutions of a two dimensional dam break problem. Int J Eng Sci 29:1357-1362

8. Glaister P (1988) Approximate Riemann solutions of the shallow water equations. J Hydraul Res 26:293-300

9. Luigi F, Toro EF (1995) Experimental and numerical assessment of the shallow water model for two dimensional dam-break problems. J Hydraul Res 33:843-864

10. Zoppou C, Roberts S (2000) Numerical solution of the two-dimensional unsteady dam break. Appl Math Fluids Res 24:457-475

11. Brufau P, Garcia-Navarro P (2000) Two dimensional dam break flow simulation. Int J Numer Methods Fluids Res 33:55-57

12. Hunt B (1982) Asymptotic solution for dam break problem. J Hydraul Div ASCE 108:115-126

13. Penney WG, Thornhill CK (1952) The dispersion, under gravity, of a column of fluid supported on a rigid horizontal plane. Phil Trans Roy Soc London A 244:285-311

14. Needham DJ, Billingham J, King AC (2007) The initial development of a jet caused by fluid, body and free surface interaction. Part 2. An impulsively moved plate. J Fluid Mech 578:67-84

15. Korobkin AA (2002) Gravity driven flows. Presentation at the BAMC (unpublished)

16. Needham DJ, Chamberlain PG, Billingham J (2007) The initial development of a jet caused by fluid, body and free surface interaction. Part 3. An inclined accelerating plate. Q J Mech Appl Math (to appear)

17. Gakhov FD (1966) Boundary value problems. Pergamon Press, Oxford 